\title{
Genomic evidence for the retention of the essential mycobacterial cell wall in the otherwise defective Mycobacterium leprae
}

\author{
PATRICK J. BRENNAN \& VARALAKSHMI D. VISSA \\ Department of Microbiology, Colorado State University, \\ Fort Collins, CO 80523, USA
}

\begin{abstract}
Summary The obligate intracellularism of Mycobacterium leprae may be attributable to the effects of mutations in major metabolic areas due to a genome capable of encoding only about 1600 proteins. Yet cell wall biosynthesis capability remains relatively intact and comparisons with the genome of Mycobacterium tuberculosis provide insights into the genetic basis of a minimal mycobacterial cell wall.
\end{abstract}

\begin{abstract}
Abbreviations
ACP:acyl carrier protein; AG: arabinogalactan; Araf: arabinofuranose; $\mathrm{C}_{50}-\mathrm{P}$ : decaprenyl 5-phosphate; CoA: coenzyme A; DAP: meso-diaminopimelic acid; DMAPP: dimethylallyl diphosphate; DPA: decaprenyl phosphoarabinose; DXP: deoxyxylulose 5-phosphate; FPP: farnesyl diphosphate; Galf: galactofuranose; GAP: glyceraldehyde 3-phosphate; GDP: guanosine 5' diphosphate; GlcNAc: $N$-acetyl glucosamine; GPP: geranyl diphosphate; IPP: isopentenyl diphosphate; LAM: lipoarabinomannan; LM: lipomannan; mAGP: mycolyl-arabinogalactan-peptidoglycan; Mal: malonyl; Man: mannose; Me: methyl; MurNAc: $N$-acetylmuramic acid; MurNGly: $N$-glycolylmuramic acid; P: phosphate; PG: peptidoglycan; PDIM: phthiocerol dimycocerosate; PGL: phenolic glycolipids; PIMs: phosphoinositol mannosides; PAPP: 5' phospho arabinofuranosyl pyrophosphate; PRPP: 5' phospho ribosyl pyrophosphate; Rha: rhamnose; TDM: trehalose dimycolate; TDP: thymidine 5'-diphosphate; TMM: trehalose monomycolate; UDP: uridine 5'-diphosphate
\end{abstract}

\section{Introduction}

In the last decade of biological research dominated by the pursuit of genomic sequence of organisms, the genus Mycobacterium has not been left behind. In fact, several species of mycobacteria have been or are currently being sequenced to aid in the prevention and treatment of diseases such as leprosy and tuberculosis in humans and Johne's disease and tuberculosis in cattle. ${ }^{1-3}$ The sequences of the virulent $M$. tuberculosis $\mathrm{H} 37 \mathrm{Rv}$, avirulent fast growing $M$. smegmatis and that of the slow-growing $M$. leprae are a valuable data set for comparative studies on physiology and virulence of mycobacteria. Unraveling the genetics of

Correspondence: P. J. Brennan (e-mail: Patrick.Brennan@ColoState.edu) 
essential biosynthetic pathways is the preferred approach for identifying new dug targets and has already yielded several candidates. ${ }^{4}$ The sequencing of the $M$. leprae bacterium is especially significant to medicine because it gives the genetic blue print of an organism yet to be cultivated in a laboratory. The sequence has revealed 'a decaying genome' with a dramatic loss of functional capacity by mutations that gave rise to 1116 pseudogenes in every aspect of central and intermediary metabolism resulting in the obligate in vivo and intracellular habitat of $M$. leprae ${ }^{1,5}$ (see also 'The decaying genome of Mycobacterium leprae', this issue). The very long doubling time of $11-12$ days $^{6}$ and the preference for a cooler environment $\left(30^{\circ} \mathrm{C}\right)^{7}$ may also be due the presence of temperature sensitive mutations in some of the remaining 1600 or so genes. Nevertheless, $M$. leprae can sustain growth and cause disease. The survival of mycobacteria under unfavorable conditions has been attributed to the characteristic permeability barrier of the cell envelope and its role in infection of host macrophages and modulation of immune responses. ${ }^{8}$ The properties of the cell envelope are particularly relevant for $M$. leprae, because the organism may depend on the host for several nutrients due to defects in the synthesis of methionine, cysteine, purine rings, and uptake of several amino acids, ions and sugars ${ }^{1,5}$ (see also 'The microbial physiologist's guide to the leprosy genome', this issue). In this report we have therefore analyzed the impact of the genome down sizing and decay on the cell wall of M. leprae. We have compared the genomes of M. leprae and M. tuberculosis and assume that genes for the biosynthesis of similar molecules are orthologous (same gene in different genomes) or at least homologous (similar gene) and those genes that are present in M. tuberculosis, but absent or pseudogenes in M. leprae are probably not involved in these syntheses or are redundant (spare).

We refer the readers to the reviews by Brennan and Nikaido ${ }^{8}$, McNeil et al. ${ }^{9}$, Chatterjee and $\mathrm{Khoo}^{10}$, Barry et al. ${ }^{11}$ Baulard et al. ${ }^{12}$, Belanger and Inamine ${ }^{13}$ and Crick et al. ${ }^{14}$ for details of the structure, function, biosynthesis and distribution among mycobacteria of these envelope components and only provide a brief outline in this review. Instead we have focused primarily on gene assignments for $M$. leprae in relation to those for $M$. tuberculosis based on the completed and annotated genome sequences.

\section{Morphology of $M$. leprae and the ultrastructure of the cell wall}

M. leprae is a strongly acid fast staining rod $1-8 \mu \mathrm{m}$ long and $0.3 \mu \mathrm{m}$ in diameter and thus does not differ remarkably from $M$. tuberculosis. Studies of the ultrastructure of $M$. leprae, in sections and as whole bacteria from man, mouse and armadillo, have been extensive ${ }^{15}$, but have not shown any gross unique features compared to other mycobacteria. However, Draper ${ }^{16}$ has described three ultrastructural features of the cell walls that may be characteristic of M. leprae: aberrant morphology, wall bands and paracrystalline bodies. He had observed departure from the classical cylindrical shape of a bacillus in suspensions prepared from armadillos, in that cells typically have a tapered or double-tapered shape with hemispherical ends, which he attributed to a defect in the normal process of cell wall construction. Wall bands first observed by Nishiura et al. are described as 'circumferential ridges on the outer surface of the cell', very numerous and positioned at random along the length of the cell. ${ }^{17}$ These may be scars left when the cell wall separated during the division process and their randomness may reflect a defect in the cell wall construction. The paracrystalline, quasi-crystalline bodies seen in sectioned $M$. leprae ${ }^{18}$ probably correspond to the capsular matrices and foamy structures responsible for binding hundreds of bacilli into 
'clumps' or globi and into smaller clumps where the individual cells occur in parallel arrays, the noted 'bundles of cigars'.

\section{Biochemical structure and composition}

Current knowledge on the biosynthesis and genetics of several components of the mycobacterial cell wall that are described below has evolved primarily from studies with M. smegmatis, $M$. tuberculosis, $M$. bovis $B C G$ and $M$. avium using a combination of methods including chemical and structural analysis, metabolic labeling, cell free assay systems, isolation and characterization of naturally occurring variants or mutants and, more recently, genetic manipulation such as mutagenesis of the genome and recombinant gene expression. However, such opportunities are limiting for $M$. leprae research due to the inability to cultivate the organism in vitro and the lack of proven genetic tools. Despite these limitations, sufficient information was gathered on the chemical and structural composition using small amounts of cells obtained from animal or human sources to conclude that the basic architecture of the cell wall is the covalently linked peptidoglycan-arabinogalactan-mycolic acids complex (mAGP) seen amongst all mycobacteria and the related corynebacteria and nocardia, except for few modifications. ${ }^{19,20}$

The peptidoglycan (PG) of M. leprae is characteristic of the chemotype IV group that includes mycobacteria, corynebacteria and nocardia because they contain meso-diaminopimelic acid (DAP), in the peptide chains. ${ }^{8}$ The muramic acids of the sugar backbone are modified with $N$-glycolyl rather than $N$-acetyl groups in mycobacteria and nocardia. ${ }^{8} \mathrm{~A}$ feature unique to $M$. leprae is the substitution of L-ala with glycine in the peptide of peptidoglycan. $^{21}$ The effect of this change on the physical properties of the peptidoglycan is not known.

Arabinogalactan (AG) is a polymer of furanose sugars of galactose and arabinose, not found in humans. ${ }^{22}$ Typically, a homogalactan ( $~ 30$ units in M. tuberculosis) composed of alternating 5 and 6 linked $\beta \mathrm{D}-\mathrm{Gal}_{f}$ residues is linked to the peptidoglycan via a disaccharide bridge (-L-Rha-D-GlcNAc-P-) called the linker unit (LU). ${ }^{23}$ Three branches of 5-linked arabinan are attached near the reducing end of the galactan. The arabinan is composed of 5-linked $\mathrm{Ara}_{f}$, which further branch (3- and 5-linked $\mathrm{Ara}_{f}-\alpha$ ). The non reducing ends are

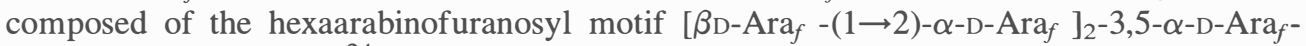
$(1 \rightarrow 5)-\alpha-\mathrm{D}-\mathrm{Ara}_{f}\left(\mathrm{Ara}_{6}\right){ }^{24}$ All these major motifs are identical in $M$. tuberculosis and $M$. leprae, with the exception that $M$. leprae has $40-50 \%$ fewer galactan residues. ${ }^{20}$ Two- $^{-}$ thirds of the terminal arabinoses of the arabinan chains are esterified with mycolic acids in M. tuberculosis. ${ }^{25}$ The extent in M. leprae has not been determined.

The $\alpha$-alkyl branch of the $\alpha$-alkyl, $\beta$-hydroxy fatty acids called mycolic acids which range from $\mathrm{C}_{14}$ to $\mathrm{C}_{26}$, is $\mathrm{C}_{20}$ in $M$. leprae. ${ }^{11}$ The $\beta$-hydroxy (meromycolate) chain is of ten modified with double bonds (cis and trans), cyclopropane, methyl, epoxy, keto, and methoxy groups that render flexibility (fluidity) to the wall. M. leprae does not have methoxymycolates ${ }^{26}$ due to the lack of a functional mmaA3 gene, as demonstrated in Mycobacterium bovis BCG (Pasteur). ${ }^{27}$ It appears that ketomycolates have a more specific role for growth in macrophages in M. tuberculosis as their abundance increases 5-fold in vivo, and under low oxygen tensions in vitro while the methoxymycolates decrease 2 -fold. The absence of ketomycolates reduces ability to survive in macrophage like cell lines. ${ }^{28}$ Therefore, the lack of methoxymycolates in $M$. leprae may not impair viability in macrophages. 
In addition to the mycolates esterified to $\mathrm{AG}$, mycolic acids are also present in the extractable lipids as esters of trehalose: 6-O-mycolyl and 6, 6'-O-dimycolyl trehalose (TMM and TDM respectively). Small amounts of TMM but not TDM were identified in M. leprae. ${ }^{29}$

The wall of $M$. leprae is also endowed with an unusually abundant proportion of the extractable intercalated lipoglycans, phosphatidylinositol mannosides (PIMs), lipomannans (LM) and lipoarabinomannans (LAM) characterized in several mycobacteria which may be anchored in the plasma membrane via the acyl chains (tuberculostearic and palmitic acid) of phosphatidyl inositol (PI). ${ }^{30}$ LM and LAM are made up of linear $\alpha-1 \rightarrow 6$ linked mannan chain originating from $\mathrm{PIM}_{2}$ in which each mannose is further branched with $\alpha-1 \rightarrow 2$ D-mannose. ${ }^{31}$ LAM is a heterogeneous macromolecule arising from LM that contains arabinan branches similar in composition and structure to that of AG that may be terminated with 'caps' of variable numbers of mannose or inositol residues. ${ }^{10,32}$ The LAM of M. leprae has fewer Ara 6 termini, and a lesser degree of mannose capping than that of $M$. tuberculosis.

However, there are other solvent extractable components ${ }^{8}$ such as glycopeptidolipids (GPLs) typical of $M$. avium spp that define serovar specificity and colony morphology; trehalose based lipids such as acylated trehaloses (containing straight chain, mycerocerosic acids, mycolipanolic and mycolipenic fatty acids) and sulpholipids (trehalose 2' sulphate acylated with phthioceranic and hydroxyphthioceranic acids) present in strains of $M$. tuberculosis; and lipooligosaccharides (LOSs) isolated from several species are absent in M. leprae. Instead, the dominant lipid is the phenolic glycolipid PGL-1 ${ }^{33}$, a glycosylated derivative of the phenolphthiocerol dimycocerosate. The trisaccharide-of PGL-1 of $M$. leprae is $\beta$-D-3, 6, di- $O$-methyl Glu $(1 \rightarrow 4)-\alpha$-2,3-di- $O$-methyl-L-Rha- $(1 \rightarrow 2)-\alpha-3-O$-methyl-L-Rha and is highly antigenic. Synthetic glycoconjugates containing this trisaccharide are sensitive tools for serodiagnostics of leprosy. ${ }^{34}$ Recently the trisaccharide was shown to be involved in the specific interaction of $M$. leprae with the laminin of Schwann cells. ${ }^{35}$ This discovery is an important step towards the identification of a mechanism for entry of $M$. leprae into nerve cells that can initiate the subsequent nerve damage that is the hallmark of leprosy. $M$. tuberculosis $\mathrm{H} 37 \mathrm{Rv}$ contains only phthiocerol dimycocerosates. ${ }^{8}$

\section{Biosynthesis and genetics of cell wall (envelope) in M. leprae: insights from the genome sequence}

The biosynthetic pathway for the individual components and their assembly to form the mAGP complex, as deciphered from the approaches mentioned before, is depicted in Figure 1. In the recent review by Crick et al. ${ }^{14}$, only the $13 \mathrm{M}$. tuberculosis genes for AGP synthesis that have been functionally characterized have been highlighted. Eleven of these were identified by the 'cloning by homology' approach. In Table 1, we show the homologs for these genes in M. leprae. We predict that these genes will be functional in $M$. leprae without the need for their functional characterization as the homology is high, and genetic context is similar. Furthermore, we have included putative genes for reactions not yet characterized in mycobacteria, by finding homologs for known genes in other organisms using the BLAST algorithm. ${ }^{36}$ The genes for the mycolic acid and phenolphthiocerol dimycocerosate synthesis are also included. In the following section, some of the biosynthetic pathways are described in brief. The common names of the genes involved are indicated in italic font in parenthesis. 


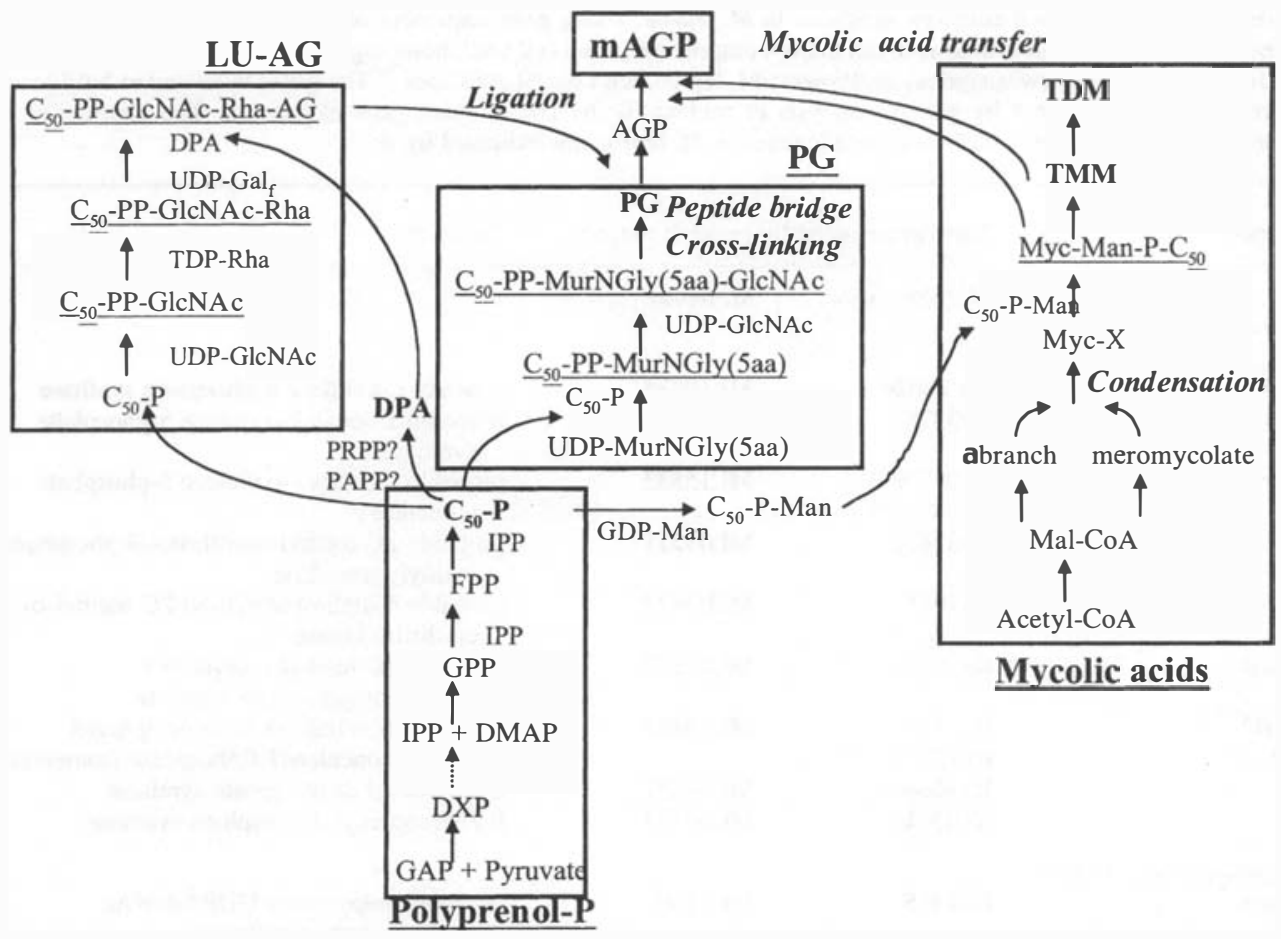

Figure 1. Biosynthesis of the mycolic acid-arabinogalactan-peptidoglycan complex (mAGP) of the cell wall of mycobacteria. The pathways for the synthesis of the individual components and the stages at which they are assembled to form the mAGP complex are shown schematically. The genes involved in these reactions and in the synthesis of the sugar donors are listed in Table 1. Polyprenyl-phosphate linked biosynthetic intermediates have been underlined. 5aa represents the pentapeptide linked to MurNGly in PG synthesis. The Myc-X represents mycolic acid esterified to an unknown carrier. Myc-Man-P-C 50 is a polyprenyl-phosphate linked mycolate, shown to be involved in transport of mycolic acids through the membrane for deposition on AG (Besra et al, Proc Natl Acad Sci USA, 1994, 91: 12735-12739).

\section{PEPTIDOGLYCAN}

The basic peptidoglycan biosynthetic machinery is similar to $E$. coli and appears to be remarkably well preserved in $M$. tuberculosis ${ }^{13,14}$ and $M$. leprae. The main events are the synthesis of the unusual sugar-nucleotide UDP-MurNAc from UDP-GlcNAc (murA, murB), the sequential addition of five amino acids to the MurNAc (murC, murD, murE and murF), transfer of the sugar-pentapeptide to a polyprenyl-phosphate carrier (murX), followed by attachment of GlcNAC from UDP-GlcNAc (murG). Glycan chains are formed by transglycosylation reactions of the disaccharide-pentapeptide chains (releasing the lipid carrier from the incoming unit), followed by cross-linking of such chains via DAP-DAP and DAP-Dala bridges in the final stage of peptidoglycan synthesis. The enzymes for transglycosylation and transpeptidation are members of the penicillin-binding protein (PBP) family. Two $M$. leprae genes (ponA and pon 1) have been cloned and expressed but their role in peptidoglycan synthesis has not been reported. ${ }^{37,38}$ The $M$. leprae genome also contains several pseudogenes with homology to PBPs. In mycobacteria, amidation of the carboxyl side chains of glutamate and DAP and the oxidation of the $N$-acetyl group of MurNAc to a glycolyl moiety occur at an undetermined stage in this pathway. 
Table 1. Genetics of cell envelope synthesis in M. leprae. Using gene sequences of the M. tuberculosis or E. coli genes involved in the biosynthesis of the major components of the cell wall, homologs were identified in the genome of M. leprae (http://www. sanger.ac.uk/Projects/M_leprae) with the BLAST tool. ${ }^{36}$ The genes indicated in bold have been characterized either by genetic analysis of mutants or by recombinant gene expression. The absence of a homolog is indicated by a dash (-). Pseudogenes in M. leprae are indicated by $\psi$

\begin{tabular}{|c|c|c|c|}
\hline Name & \multicolumn{2}{|c|}{ Number assigned by genome project } & \multirow[t]{2}{*}{ Function } \\
\hline & M. tuberculosis & M. leprae & \\
\hline \multicolumn{4}{|c|}{ Polyprenyl-P synthesis } \\
\hline$d x s-I$ & Rv 2682c & ML10388 & 1-deoxy-D-xylulose 5-phosphate synthase \\
\hline$d x s-I I$ & Rv3379c & - & $\begin{array}{l}\text { probable 1-deoxy-D-xylulose 5-phosphate } \\
\text { synthase }\end{array}$ \\
\hline$d x r$ & Rv2870c & ML15833 & $\begin{array}{l}\text { probable 1-deoxy-D-xylulose 5-phosphate } \\
\text { reductase }\end{array}$ \\
\hline$y g b P$ & Rv3582c & ML03211 & $\begin{array}{l}\text { probable } 2 \mathrm{C} \text {-methyl-D-erithritol-4-phosphate } \\
\text { cytidylyltransferase }\end{array}$ \\
\hline$y \operatorname{ch} B$ & Rv1011 & ML02422 & $\begin{array}{l}\text { probable 4-diphosphocytidyl-2C-methyl-D- } \\
\text { erythritol kinase }\end{array}$ \\
\hline$y g b B$ & Rv 3581c & ML03222 & $\begin{array}{l}\text { probable } 2 \mathrm{C} \text {-methyl-D-erythritol } \\
2,4 \text {-cyclodiphosphate synthase }\end{array}$ \\
\hline lyt B2 & Rv1110 & ML19388 & gene function has not been deciphered \\
\hline \multirow[t]{3}{*}{ idi } & Rv1745c & - & probable isopentenyl diphosphate isomerase \\
\hline & Rv1086 & ML24677 & $\mathrm{E}, \mathrm{Z}$ farnesyl disphosphate synthase \\
\hline & Rv2361c & ML06344 & E,Z decaprenyl diphosphate synthase \\
\hline \multicolumn{4}{|c|}{ Peptidoglycan synthesis } \\
\hline murA & Rv1315 & ML1150 & $\begin{array}{l}\text { phosphoenolpyruvate:UDP-GlcNAc } \\
\text { enolypyruvate transferase }\end{array}$ \\
\hline $\operatorname{mur} B$ & Rv0482 & ML2447 & $\begin{array}{l}\text { UDP- } N \text {-acetylenolpyruvoylglucosamine } \\
\text { reductase }\end{array}$ \\
\hline murC & Rv2152c & ML0915 & UDP-MurNAC: L-alanine ligase \\
\hline $\operatorname{mur} D$ & Rv2155c & ML0912 & UDP-MurNAc-L-ala: D-glutamate ligase \\
\hline murE & $\mathrm{Rv} 2158 \mathrm{c}$ & ML0909 & UDP- $N$-acetylmuramyl-tripeptide synthetase \\
\hline $\operatorname{mur} F$ & $\operatorname{Rv} 2157 c$ & ML0910 & UDP-MurNAc-pentapeptide synthetase \\
\hline murX & Rv2156c & ML0911 & UDP-acetylmuramyl-tripeptide synthetase \\
\hline $\operatorname{mur} G$ & Rv2153 & ML0915 & $\begin{array}{l}\text { pentapeptide pyrophosphoryl-decaprenol } \\
N \text {-GlcNAc transferase }\end{array}$ \\
\hline ponA & Rv0050 & ML2688 & PBP; transpeptidase or transglycosylase \\
\hline pon $A^{\prime}$ & Rv3682 & ML2308 & PBP; transpeptidase or transglycosylase \\
\hline \multicolumn{4}{|c|}{$\begin{array}{l}\text { Linker unit-arabinogalactan synthesis } \\
\text { dTDP-rhamnose synthesis }\end{array}$} \\
\hline$r m l A$ & Rv0334 & ML2503 & $\begin{array}{l}\text { D-glucose } 1 \text {-phosphate thymidylyl } \\
\text { transferase }\end{array}$ \\
\hline$r m l B$ & Rv3464 & ML1964 & dTDP-D-glucose-4,6 dehydratase \\
\hline$r m l C$ & Rv3465 & ML1965 & dTDP-4-dehydrorhamnose 3,5-epimerase \\
\hline$r m l D$ & Rv3266c & ML0751 & dTDP-4-dehydro-rhamnose reductase \\
\hline \multicolumn{4}{|c|}{ UDP-galactofuranose synthesis } \\
\hline galE & Rv3634c & ML0204 & UDP-glucose-4-epimerase \\
\hline glf & Rv3809c & ML0092 & UDP-galactopyranose mutase \\
\hline \multicolumn{4}{|c|}{ Lipid linked linker unit-arabinogalactan polymerization } \\
\hline$r f e$ & Rv1302 & ML1137 & $\begin{array}{l}\text { probable UDP-GlcNAc: } \mathrm{C}_{50}-\mathrm{P} \text { GlcNAc } \\
\text { transferase }\end{array}$ \\
\hline$w b b l$ & Rv3265c & ML0752 & $\begin{array}{l}\text { probable dTDP-rhamnose: } \mathrm{C}_{50} \text {-PP-GlcNAc } \\
\text { rhamnose transferase }\end{array}$ \\
\hline glfT & Rv3808c & ML0093 & UDP-galactofuranose transferase \\
\hline$e m b C$ & Rv3793 & ML0106 & $\begin{array}{l}\text { arabinofuranose transferase? (arabinan } \\
\text { synthesis) }\end{array}$ \\
\hline$e m b A$ & Rv3794 & ML0105 & \\
\hline$e m b B$ & Rv3795 & ML0104 & \\
\hline
\end{tabular}


Table 1. Continued

Name Number assigned by genome project Function

Mycolic acid synthesis and deposition $\alpha$-branch synthesis

fas Rv2524c

Meromycolic acid synthesis

$\operatorname{accD6}$

Rv2247

acpM

Rv2244

$f a b D$

Rv2243

fabH

Rv0533c

kasA

Rv2245

kas $B$

Rv2246

$m a b A$

Rv1483

inhA

Rv1484

Meromycolic acid modification

cmaAl

Rv3392c

cmaA2

Rv0503c

mmaAl

Rv0645c

Rv0644c

$\operatorname{mmaA2}$

Rv0643c

mmaA3

Rv0642c

mmaA4

Rv0469

umaA2 (pcaA)

Rv0470c

$\begin{array}{ll}\operatorname{des} A 1 & \mathrm{Rv} 0824 \mathrm{c} \\ \operatorname{des} A 2 & \mathrm{Rv} 1094 \\ \operatorname{des} A 3 & \mathrm{Rv} 3229 \mathrm{c}\end{array}$

Deposition of mycolic acids

$f b p A$

Rv3804c

$\begin{array}{ll}f b p B & \text { Rv1886c } \\ f b p C 2 & \mathbf{R v 0 1 2 9 c}\end{array}$

PGL-1 synthesis

Mycocerosoic acid synthesis

mas

Rv2940c

fadD28

Rv2941

mmpL7

Rv2942

ML0139

ML0138

ML0137

Phthiocerol synthesis

$\begin{array}{ll}\text { fad } D 26 & \text { Rv293 } \\ \text { pps } A & \text { Rv293 } \\ \text { pps } B & \text { Rv293 } \\ \text { pps } C & \text { Rv293 } \\ \text { pps } D & \mathbf{R v 2 9 3} \\ \text { pps } E & \mathbf{R v 2 9 3} \\ d r r A & \mathbf{R v 2 9 3} \\ d r r B & \mathbf{R v 2 9 3} \\ d r r C & \mathbf{R v 2 9 3} \\ \text { pap } A 5 & \mathbf{R v 2 9 3}\end{array}$

ML2358

ML2357

ML2356

ML2355

ML2354

ML2353

ML2352

ML2351

ML2350

ML2349

Glycosylation of PDIM (trisaccharide synthesis)

Rv1524

ML2348

Rv1526c

ML2348?

Rv2962c

ML0125 fatty acid synthase (FASI)

acetyl-CoA carboxylase (malonyl-CoA

synthase)

acyl carrier protein

malonyl-CoA-[ACP]-transacylase

(malonyl-ACP) synthase

$\beta$-ketoacyl-ACP synthase III

$\beta$-ketoacyl-ACP synthase

3-ketoacyl-ACP reductase

enoyl-ACP reductase

cyclopropane mycolic acid synthase (distal)

cyclopropane mycolic acid synthase (proximal)

trans cyclopropane mycolic acid synthase (oxygenated mycolates)

cyclopropane mycolic acid synthase (oxygenated mycolates)

methoxymycolate synthase

hydroxymycolate synthase

probable mycolic acid methyltransferase

cyclopropane mycolic acid synthase (proximal, $\alpha$-mycolates)

probable acyl-ACP desaturase

mycolyltransferase (TMM, TDM and mAGP synthesis?)

mycocerosoic acid synthase

probable acyl-CoA synthase

mycobacterium membrane protein

(transport of PDIM)

probable acyl-CoA synthase

(phenol) phthiocerol synthase

"

$"$

$"$

"

?

?

transport of PDIM

polyketide associated protein (transport of PDIM)

probable TDP-Rhamnose:phenol PDIM rhamnose transferase

probable UDP-glucose: phenol

PDIM-rhamnose glucose transferase 
Table 1. Continued

\begin{tabular}{|c|c|c|c|}
\hline \multirow[t]{2}{*}{ Name } & \multicolumn{2}{|c|}{ Number assigned by genome project } & \multirow{2}{*}{$\begin{array}{l}\text { Function } \\
\text { " }\end{array}$} \\
\hline & Rv2958c & ML0128 & \\
\hline & Rv2959c & ML0127 & probable methyltransferase \\
\hline & Rv2952 & ML0130 & probable methyltransferase \\
\hline \multicolumn{4}{|c|}{ PIMs, LM and LAM synthesis } \\
\hline & Rv0486 & ML2443 & $\begin{array}{l}\text { probable GDP-mannose: polyprenyl- } \\
\text { P mannosyl transferase }\end{array}$ \\
\hline & Rv2051c & ML1440 & $"$ \\
\hline \multirow[t]{3}{*}{$\operatorname{pgs} A$} & Rv2612c & ML0454 & $\begin{array}{l}\text { CDP-diacylglycerol: inositol phosphitidyl } \\
\text { transferase }\end{array}$ \\
\hline & Rv2611c & ML0452 & $\begin{array}{l}\text { probable phosphitidylinositol: } \\
\text { GDP-mannose mannose transferase }\end{array}$ \\
\hline & Rv2610c & ML0453 & $\begin{array}{l}\text { probable diacylphosphitidylinositol } \\
\text { mannose: palmitoyl-CoA acyltransferase }\end{array}$ \\
\hline \multirow[t]{4}{*}{$\operatorname{pim} B$} & Rv0557 & ML2272 $\psi$ & $\begin{array}{l}\text { triacylphosphitidylinositol mannose: } \\
\text { GDP-mannose mannosyl transferase }\end{array}$ \\
\hline & Rv2188c & ML0886 & $\begin{array}{l}\text { probable mannose transferase } \\
\text { (mannan synthesis?) }\end{array}$ \\
\hline & Rv3032 & ML1715 & $"$ \\
\hline & $\mathrm{Rv} 0225$ & ML2583 & $"$ \\
\hline
\end{tabular}

Since the M. leprae peptidoglycan has glycine rather than L-alanine in the peptide crosslinks, it was thought there might be a genetic basis for this substitution. Mahapatra et al. ${ }^{39}$ analysed the genome but could not find a second ligase gene. In fact they demonstrated by in vitro studies with the recombinant MurC enzyme, that it can use L-alanine or glycine substrate with comparable affinities. Therefore the presence of glycine in $M$. leprae is perhaps an in vivo phenomenon driven by the ambient amino acid milieu.

\section{ARABINOGALACTAN}

The synthesis of AG requires the sugar donors UDP-GlcNAc, ${ }^{40}$ TDP-rhamnose ( $r m l A, r m l B$, $r m l C$ and $m l D)^{40,41}$, UDP-galactofuranose UDP-gal ${ }_{f}(\text { galE and } g l f)^{42}$ and decaprenylphospho-arabinose DPA. ${ }^{43}$ Successive addition of GlcNAc $(r f e)$, rhamnose $(w b b l)$, galactofuranose and arabinofuranose on a prenyl-phosphate lipid carrier ${ }^{44}$ occurs before the entire LU-AG is transferred (ligated) to approximately 1 in 10 MurNAc units of peptidoglycan.

The synthesis of DPA is interesting and is proposed to originate from the pentose phosphate pathway as phosphoribose pyrophosphate (PRPP). ${ }^{45}$ Epimerization of the ribose to arabinose may occur before or after transfer to a decaprenyl-phosphate carrier. Regarding the galactosyltransferases, Mikusova et al. ${ }^{44}$ showed that the gene Rv3808c ( $\mathrm{glfT}$ ) of $M$. tuberculosis, is a galactosyltransferase in AG synthesis. There is an ortholog in M. leprae. Furthermore, Kremer et al. ${ }^{46}$ suggest that it encodes a bi-functional transferase for the alternating 5 and 6 linked galactose residues of the galactan by use of synthetic acceptors. It has not been demonstrated if GlfT can also catalyse the addition of the first galactose unit to the rhamnose sugar of the linker unit and the second galactose of the galactan. GlfT contains the sugar nucleotide binding motif hhhhDxDxh where ' $h$ ' represents an amino acid with hydrophobic nature. 
With regard to arabinosyltransferases, the work of Belanger ${ }^{47}$ suggests that the embA or $e m b B$ genes of $M$. avium encode putative transferases for AG. A third gene, $e m b C$, also exists in all mycobacteria sequenced thus far and Escuyer et al. ${ }^{48}$ have shown that knocking out the embA or embB genes of $M$. smegmatis causes changes in the arabinan content and structure of AG. These Emb proteins are very homologous to each other; they are large and hydrophobic, and span membranes. The genes are well conserved amongst many mycobacteria and are intact in $M$. leprae in a gene cluster very similar to that seen in M. tuberculosis. However, the proteins they encode have no significant homology to any other proteins in the database and no known domains or motifs. It is not clear if the Emb proteins are involved in the actual glycosyltransferase catalysis or in the assembly of the arabinan.

PRENYL-PHOSPHATE CARRIERS

The biosynthesis of many of the key cell wall polymers requires prenyl-phosphate carriers as sugar donors (DPM and DPA) and for carriers of the intermediates of cell wall polysaccharide synthesis (AG and PG). ${ }^{14}$ The synthesis begins with the formation of deoxyxylulose phosphate from pyruvate and glyceraldehydes-3P $(d x s-I)$, which is converted to the 5carbon isoprene compounds isopentenyl pyrophosphate (IPP) and its isomer dimethylallyl pyrophosphate (DMAPP) by the non-mevalonate pathway, also called the 2-C-methyl-Derythritol 4-phosphate (MEP) pathway. ${ }^{49}$ In M. tuberculosis there are two possible genes for this function $(d x s-I$ and $d x s-I I)$. DXS-I has been shown to be functional by expression in E. coli. ${ }^{50}$ Studies on DXS-II, are in progress. However, $d x s-I I$ may be redundant in $M$. tuberculosis, since the $M$. leprae genome has only one gene that is homologous to $d x s$ I. The complete pathway for the synthesis of IPP and DMAPP is not known. However, for all genes identified in $E$. coli ${ }^{51,52}$, homologous genes have been found in M. tuberculosis and $M$. leprae for this essential pathway. A non-essential IPP isomerase (idi) for the interconversion of IPP and DMAPP is present in E. coli ${ }^{53}$ and M. tuberculosis but not in $M$. leprae. The gene responsible for condensation of IPP with DMAP, to form geranyl diphosphate (GPP, $\mathrm{C}_{10}$-PP) has not been identified in any organism. The addition of IPP to GPP results in farnesyl diphosphate (FPP, $\mathrm{C}_{15}-\mathrm{PP}$ ), which is subsequently elongated by seven cycles of polymerization to form decaprenyl diphosphate in a specific stereochemistry in $M$. tuberculosi ${ }^{54}$ and also probably in $M$. leprae because homologous polymerase genes exist. In terms of synthesis of other isoprenoids, there are no homologs in M. leprae for four other prenyldiphosphate synthase genes found in the M. tuberculosis genome, except for the $\mathrm{grcCl}$ gene, which may be involved in the transfer of a prenyl moiety in the menaquinone pathway. There are also no homologs in M. leprae for the M. tuberculosis squalene synthase, monoxygenase and cyclase genes probably involved in steroid synthesis.

\section{Biosynthesis of PI, PIMs, LM and LAM}

The biosynthesis of phosphatidylinositol mannosides is initiated on the precursor PI using the gene product of $p g s A^{55}$, followed by mannosylation using GDP-mannose as the sugar donor. ${ }^{56}$ Genes for a mannosyltransferase and an acyltransferase are linked to pgs $A$ and may be required for the synthesis of $\mathrm{PIM}_{1}$ and the acylation of mannose to form triacyl $\mathrm{PIM}_{1}$, respectively. The gene product of Rv0557 (pimB) of M. tuberculosis has been identified as the second mannosyltransferase. ${ }^{57}$ The rPimB was shown to convert labeled 
tri-acylated PIM $_{1}$ to PIM $_{2}$ in the presence of GDP-mannose. PimB belongs to the family of glycosyltransferases that have a conserved $\mathrm{C}$ terminal motif $\mathrm{EX}_{7} \mathrm{E}^{58}$ This gene is a pseudogene in $M$. leprae and we predict that one of the other genes sharing a homologous active site is used (Table 1). Subsequent mannosylation with undefined enzymes generates LM.

Of the many putative glycosyltransferases in the $M$. tuberculosis genome, several (Rv0539, Rv0696, Rv1781c, Rv1500, Rv1513, Rv1514c, Rv1516c, Rv1518, Rv1520 and Rv1525) do not have orthologs in M. leprae. We propose that these enzymes are not involved in the biosynthesis of mannan, arabinan and galactan, since there are no significant structural differences between these molecules in M. tuberculosis and M. leprae.

\section{Mycolic acids, TMM and TDM and deposition on AG}

The synthesis of mycolates occurs in several stages: synthesis of the $\alpha$-alkyl chain and the primer for the meromycolic acid by the multifunctional fatty acyl synthase FASI enzyme $(f a s)^{59}$; linking by the $\beta$-keto acyl synthase III $(f a b H)^{60}$ and elongation of the primer ${ }^{11}$ by the disassociated fatty acyl synthase complex FASII (accD6, fabD, kasA, kasB, acpM, mabA, $\operatorname{inh} A) ;{ }^{61,62}$ modification of the meromycolic acid (introduction of double bonds, cyclopropane rings, keto, methyl and methoxy groups), probably in parallel with elongation; ${ }^{63}$ and finally condensation of the $\alpha$-alkyl chain and meromycolate. The condensation step and the carrier on which this reaction occurs are not known. The mycolates are then transferred to AG and trehalose to form mAGP and TMM/TDM respectively and may occur using the mycolyltransferases encoded by the members of the antigen 85 complex $(f b p A, f b p B$ and $f b p C 2)^{64,65}$. All of these steps have been characterized in M. tuberculosis and homologs for the genes are present in $M$. leprae, with the exception of the gene encoding the linking enzyme FabH. The mechanism in M. leprae is not clear.

\section{Phenolic glycolipids PGLs (glycosylphenolpthiocerol dimycocerosates)}

The phthiocerol moiety is synthesized using a set of multifunctional enzymes ( $p p s A$, pps $B$, pps , pps $D$ and ppsE ) that contain one or more of the acyltransferase, ketoacyl synthase, keto reductase, dehydratase, enoyl reductase and acyl carrier modules for the polymerization of malonyl-CoA and methylmalonyl-CoA units on a $\mathrm{C}_{22}-\mathrm{CoA}$ fatty acid precursor. ${ }^{66}$ Mycocerosic acid syntheisis occurs by the elongation of fatty acyl-CoA primers with methylmalonyl-CoA ${ }^{67}$ (mas), followed by the transfer to the phthiocerol using a specific acyl-CoA synthase ( $f a d D 28)$. Two membrane associated proteins MmpL7 and DrrC have been shown to be responsible for the transport of the PDIM. In M. tuberculosis, genes for all these functions are clustered on the genome and mutations in these genes result in disruption of PDIM synthesis and loss of virulence. ${ }^{68}$ In $M$. leprae, the pps $A-E$ genes are intact but have been separated from the mas, fadD28 and $m m p L 7$ genes. For the addition of the first rhamnose in the trisaccharide of PGL-1 in $M$. leprae, we have analyzed the genome for genes homologous to the rhamnosyltransferases such as $r t f A$ of $M$. avium (for addition of Lrhamnose to the 6-deoxy talose in GPL synthesis ${ }^{69}$ ), and the $w b b L$ gene of $M$. tuberculosis (involved in linker unit synthesis). Based on homology searches with $r t f A$, we have 
identified the gene ML2348 in M. leprae as a candidate. Co-incidentally, ML2348 is located where the phthiocerol gene cluster of $p p s / d r r / p a p A 5$ has separated from the mas/fadD28/ $m m p L 7$ in $M$. leprae and may indicate its role in PGL-1. ML2348 is also homologous to genes used for the synthesis of glycosylated steroids in plants, and for antibiotics such as balhimycin and tylosin (tylN, 6-deoxyallosyltransferase) in microbes. ${ }^{70}$ All these homologs use sterol/phenol like acceptors. Furthermore, $r t f A$ and tyl $l N$ encode glycosyltransferases for sugars other than glucose (particularly 6-deoxy hexoses). Combining these pieces of information, we propose that ML2348 is a good candidate for the enzyme that transfers the first rhamnose. We postulate that the genes ML0125 and ML0128 are glycosyltransferases and ML0127 and ML0130 are methylases for the synthesis of the second and third sugars of PGL-1. These genes are located close to the mycocerosoic acid gene cluster mas/ fadD28/mmpL7 genes. Genes that are highly homologous to ML2348 are also present in $M$. tuberculosis (RV1524 and 1526c). However, these are clustered with another pks system (pks5). There are also homologs in M. tuberculosis for the candidate glycosyltransferases (Rv2958c and Rv2962c) and methyltransferases (RV 2952 and Rv2959c) in a cluster similar to that in $M$. leprae, but may have no function since only phthiocerols and not the phenolpthiocerols are found in M. tuberculosis. ${ }^{8}$

\section{Concluding remarks}

In this review, we have identified putative genes of $M$. leprae for some biosynthetic pathways by homology searches with known genes of other organisms. We are comfortable with the premise that the genome of $M$. leprae approaches a minimal and perhaps also an 'essential' gene set for all basic structural and biological properties shared by virulent and avirulent mycobacteria, particularly for cell wall core synthesis. Genes for the synthesis of precursor molecules that cannot be obtained from the host environment such as UDP-Gal ${ }_{f}$ and TDP-Rha for AG, PI for PIMs, LM and LAM and DXP for polyprenyl phosphates have been retained in M. leprae.

In terms of the practical approaches to verifying and harnessing this genetic information, recombinant proteins can be purified and used in suitable assays. In the future, it may even be possible to set up de novo synthesis of complex macromolecules in crude extracts of $M$. leprae, since viable and high titre $M$. leprae are now available from nude mouse foot pads. ${ }^{71}$ In addition, $M$. le prae from this source have been kept viable for up to 6 weeks in broth culture at $30^{\circ} \mathrm{C}$. Since the number of functional ORFs is smaller than $M$. tuberculosis, use of microarrays and comparative proteomics, is a reasonable approach to identify genes that are preferentially regulated under defined and modified test conditions in vitro. We believe the M. leprae sequence information will be valuable towards efforts for elimination of leprosy.

\section{Acknowledgements}

Research conducted in the authors' laboratory was supported by NIH, NIAID, DMID Contract NO1 AI-55262 from the National Institute of Allergy and Infectious Diseases, NIH, and by the Heiser Program for Research in Leprosy and Tuberculosis, New York City, USA. We thank Dean Crick for helpful discussions. 


\section{References}

${ }^{1}$ Cole ST, Eiglmeier K, Parkhill J et al. Massive gene decay in the leprosy bacillus. Nature, 2001; 409: 1007-1011.

2 Cole ST, Brosch R, Parkhill J et al. Deciphering the biology of Mycobacterium tuberculosis from the complete genome sequence. Nature, 1998; 393: 537-544.

3 The Mycobacterium leprae Genome Project [http://www.sanger.ac.uk/Projects/M_leprae/]; The Mycobacterium tuberculosis Genome Project [http://www.sanger.ac.uk/Projects/M_tuberculosis/]; the Mycobacterium bovis Genome Project [http://www.sanger.ac.uk/Projects/M_bovis/]; The Mycobacterium paratuberculosis Genome Project [http://www.cbc.umn.edu/ResearchProjects/AGAC/Mptb/Mptbhome.html]; The Corynebacterium diphtheriae Genome Project [http://www.sanger.ac.uk/Projects/C_diphtheriae] The Institute for Genomic Research [http://www.tigr.org].

4 Crick DC, Brennan PJ. Antituberculosis drug research. Curr Opin Anti-Infect Invest Drugs 2000; 2: 154-163.

5 Vissa VD, Brennan PJ. The genome of Mycobacterium leprae: a minimal mycobaacterial gene set. Genome Biol, 2001; 2: 1023-1030.

${ }^{6}$ Levy L. Studies of the mouse footpad technique for cultivation of Mycobaacterium leprae 3. Doubling time during logarithmic multiplication. Lepr Rev, 1976; 47: 103-106.

${ }^{7}$ Shepard CC. Stability of Mycobacterium leprae and the temperature optimum for growth. Int J Lepr, 1965; 33: 541-550.

8 Brennan PJ, Nikaido H. The envelope of mycobacteria. Annu Rev Biochem, 1995; 64: 29-63.

9 McNeil M, Besra GS, Brennan PJ. Chemistry of the mycobacaterial cell wall. In: Rom WN, Garay SM (eds) Tuberculosis. Little, Brown and Company, Boston, 1996, pp. 171-185.

10 Chatter jee D, Khoo KH. Mycobacterial lipoarabinomannan: an extraordinary lipoheteroglycan with profound physiological effects. Glycobiology, 1998; 8: 113-120.

11 Barry CE 3rd, Lee RE, Mdluli K et al. Mycolic acids: structure, biosynthesis and physiological functions. Prog Lipid Res, 1998; 37: 143-179.

12 Baulard AR, Besra GS, Brennan PJ. The cell-wall core of Mycobacterium: structure, biogenesis and genetics. In: Ratledge C, Dale J (eds) Mycobacteria, molecular biology and virulence. Blackwell Science, Oxford, 1999, pp 240-259.

13 Belanger AE, Inamine JM. Genetics of cell wall biosynthesis. In: Hatfull GF, Jacobs WR Jr (eds) Molecular genetics of mycobacteria. ASM Press, Washington DC, 2000, pp 191-202.

14 Crick DC, Mahapatra S, Brennan PJ. Biosynthesis of the arabinogalactan-peptidoglycan complex of Mycobacterium tuberculosis. Glycobiology, 2001, 11: 107R-118R.

15 Hirata T. Electron microscopic observations of cell wall and cytoplasmic membrane in murine human leprosy bacilli. Int J Lepr, 1985; 53: 433-440.

16 Draper P. The bacteriology of Mycobacterium leprae. Tubercle, 1983; 64: 43-56.

17 Nishiura M, Okada S, Izumi S, Takizawa H. An electron microscope study of the band structure of the leprosy bacillus and other mycobacteria. Int J Lepr, 1969; 37: 225-238.

18 David HL, Clavel S, Clement F, Lesourd M. Paracrystalline inclusions in Mycobacaterium leprae. Annal Microbiol (Paris), 1981; 132A: 41-50.

19 Draper P, Kandler O, Darbre A. Peptidoglycan and arabinogalactan of Mycobacterium leprae.J Gen Microbiol, 1987; 133: 1187-1194.

${ }^{20}$ Daffe M, McNeil M, Brennan PJ. Major structural features of the cell wall arabinogalactans of Mycobacterium, Rhodococcus, and Nocardia spp. Carbohydr Res, 1993; 249: 383-398.

21 Draper P. Cell walls of Mycobacterium leprae. Int J Lepr, 1976; 44: 95-98.

22 McNeil M, Wallner SJ, Hunter SW, Brennan PJ. Demonstration that the galactosyl and arabinosyl residues in the cell-wall arabinogalactan of Mycobacterium le prae and Mycobacterium tuberculosis are furanoid. Carbohydr Res, 1987; 166: 299-308.

23 McNeil M, Daffe M, Brennan PJ. Evidence for the nature of the link between the arabinogalactan and peptidoglycan of mycobacterial cell walls. J Biol Chem, 1990; 265: 18200-18206.

24 Daffe M, Brennan PJ, McNeil M. Predominant structural features of the cell wall arabinogalactan of Mycobacterium tuberculosis as revealed through characterization of oligoglycosyl alditol fragments by gas chromatography/mass spectrometry and by ${ }^{1} \mathrm{H}$ and ${ }^{13} \mathrm{C}$ NMR analyses. J Biol Chem, 1990; 265: 6734-6743.

25 McNeil M, Daffe M, Brennan PJ. Location of the mycolyl ester substituents in the cell walls of mycobacteria. $J$ Biol Chem, 1991; 266:13217-13223.

${ }^{26}$ Minnikin DE, Dobson G, Goodfellow M et al. Quantitative comparison of the mycolic and fatty acid compositions of Mycobacterium leprae and Mycobacterium gordonae. J Gen Microbiol, 1985; 131: 2013-2021.

27 Behr MA, Schroeder BG, Brinkman JN et al. A point mutation in the mma3 gene is responsible for impaired methoxymycolic acid production in Mycobacterium bovis BCG strains obtained after 1927. J Bacteriol, 2000; 182: 3394-3399.

28 Yuan Y, Zhu Y, Crane DD, Barry CE 3rd. The effect of oxygenated mycolic acid composition on cell wall function and macrophage growth in Mycobacterium tuberculosis. Mol Microbiol, 1998; 29: 1449-1458. 
29 Dhariwal KR, Yang YM, Fales HM, Goren MB. Detection of trehalose monomycolate in Mycobacterium leprae grown in armadillo tissues. J Gen Microbiol, 1987; 133: 201-209.

30 Hunter SW, Brennan PJ. Evidence for the presence of a phosphatitidylinositol anchor on the lipoarabinomannan and lipomannan of Mycobacterium tuberculosis. J Biol Chem. 1990; 265: 9272-9279.

31 Khoo KH, Dell A, Morris HR et al. Structural definition of acylated phosphatidylinositol mannosides from Mycobacterium tuberculosis: definition of a common anchor for lipomannan and lipoarabinomannan. Glycobiology, 1995; 5: 117-127.

32 Khoo KH, Dell A, Morris HR et al. Inositol phosphate capping of the nonreducing termini of lipoarabinomannan from rapidly growing strains of Mycobacterium. J Biol Chem, 1995; 270: 12380-12389.

${ }^{33}$ Hunter SW, Brennan PJ. A novel phenolic glycolipid from Mycobacterium leprae possibly involved in immunogenicity and pathogenicity. J Bacteriol, 1981; 147: 728-735.

34 Brennan PJ, Chatterjee D, Fujiwara T, Cho S-N. Leprosy specific neoglycoconjugates: synthesis and application to serodiagnosis of leprosy. Methods Enzymol, 1994; 242: 27-37.

35 Rambukkana A. Molecular basis for the peripheral nerve predilection of Mycobacterium leprae. Curr Opin Microbiol, 2001; 4: 21-27.

36 Altschul SF, Madden TL, Schäffer AA et al. Gapped BLAST and PSI-BLAST: a new generation of protein database search programs. Nucleic Acids Res, 1997; 25: 3389-3402.

37 Lepage S, Dubois P, Ghosh TK et al. Dual multimodular class A penicillin-binding proteins in Mycobacterium leprae. J Bacteriol, 1997; 179: 4627-4630.

38 Basu J, Mahapatra S, Kundu M, Mukhopadhyay S et al. Identification and overexpression in Escherichia coli of a Mycobacterium leprae gene, pon1, encoding a high-molecular-mass class A penicillin-binding protein, PBP1. J Bacteriol, 1996; 178: 1707-1711.

39 Mahapatra S, Crick DC, Brennan PJ. Comparison of the UDP-N-acetylmuramate:L-alanine ligase enzymes from Mycobacterium tuberculosis and Mycobacterium leprae. J Bacteriol, 2000; 182: 6827-6830.

40 Mikusova K, Mikus M, Besra GS et al. Biosynthesis of the linkage region of the mycobacterial cell wall. $J$ Biol Chem, 1996; 271: 7820-7828.

41 Ma Y, Stern RJ, Scherman MS et al. Drug targeting Mycobacterium tuberculosis cell wall synthesis: genetics of dTDP-rhamnose synthetic enzymes and development of a microtiter plate-based screen for inhibitors of conversion of dTDP-glucose to dTDP-rhamnose. Antimicrob Agents Chemother, 2001; 45: 1407-1416.

42 Weston A, Stern RJ, Lee RE et al. Biosynthetic origin of mycobacterial cell wall galactofuranosyl residues. Tuberc Lung Dis, 1997; 78: 123-131.

43 Wolucka BA, McNeil MR, de Hoffmann E et al. Recognition of the lipid intermediate for arabinogalactan/ arabinomannan biosyshtesis and its relation to the mode of action of ethambutol on mycobacteria. $J$ Biol Chem, 1994; 269: 23328-23335.

44 Mikusova K, Yagi T, Stern R et al. Biosynthesis of the galactan component of the mycobacterial cell wall. J Biol Chem, 2000; 275: 33890-33897.

45 Scherman MS, Kalbe-Bournonville L, Bush D et al. Polyprenylphosphate-pentoses in mycobacteria are synthesized from 5-phosphoribose pyrophosphate. J Biol Chem, 1996; 271: 29652-29658.

46 Kremer L, Dover LG, Morehouse C et al. Galactan biosynthesis in Mycobacterium tuberculosis: identification of a bifunctional UDP-galactofuranosyltransferase. J Biol Chem, 2001; 276: 26430-26440.

47 Belanger AE, Besra GS, Ford ME et al. The embAB genes of Mycobacterium avium encode an arabinosyl transferase involved in cell wall arabinan biosynthesis that is the target for the antimycobacterial drug ethambutol. Proc Natl Acad Sci USA, 1996; 93: 1919-1924.

${ }^{48}$ Escuyer VE, Lety ME, Torrelles JB et al. The role of the $e m b A, B$ gene products in the biosynthesis of the terminal hexaarabinofuranosyl motif of Mycobacterium smegmatis arabinogalactan. J Biol Chem, 2001; Oct 24.

49 Rohmer, M, Knani, M, Simonin, P et al. Isoprenoid biosynthesis in bacteria: a novel pathway for the early steps leading to isopentenyl diphosphate. Biochem. J, 1993; 295: 517-524.

50 Bailey AM, Mahapatra S, Brennan PJ, Crick DC. Purification and characterization of 1-deoxyxylulose 5phosphate synthase from Mycobacterium tuberculosis. Glycobiology, 2000; 10: 46.

51 Takahashi S, Kuzuyama T, Watanabe H, Seto H. A 1-deoxy-D-xylulose 5-phosphate reductoisomease catalyzing the formation of 2-C-methyl-D-erythritol 4-phosphate in an alternative nonmevalonate pathway for terpenoid biosynthesis. Proc Natl Acad Sci USA, 1998; 95: 9879-9884.

52 Campos N, Rodriguez-Concepcion M, Sauret-Gueto S et al. Escherichia coli engineered to synthesize isopentenyl diphosphate and dimethylallyl diphosphate from mevalonate: a novel system for the genetic analysis of the 2-Cmethyl-D-erythritol 4-phosphate pathway for isoprenoid biosynthesis. Biochem J, 2001; 353: 59-67.

53 Rodriguez-Concepcion M, Campos N, Lois LM et al. Genetic evidence of branching in the isoprenoid pathway for the production of isopentenyl diphosphate and dimethylallyldiphosphate in Escherichia coli. FEBS lett, 2000; 473: $328-332$.

54 Schulbach MC, Brennan PJ, Crick DC. Identification of a short $\left(\mathrm{C}_{15}\right)$ chain Z-isoprenyl diphosphate synthase and a homologous long $\left(\mathrm{C}_{50}\right)$ chain isoprenyl diphosphate synthase in Mycobacterium tuberculosis. J Biol Chem, 2000; 275: 22876-22881. 
55 Jackson M, Crick DC, Brennan PJ. Phosphatidylinositol is an essential phospholipid of mycobacteria. J Biol Chem, 2000; 275: 30092-30099.

56 Besra GS, Morehouse CB, Rittner CM et al. Biosynthesis of mycobacterial lipoarabinomannan. J Biol Chem, 1997; 272: 18460-18466.

57 Schaeffer ML, Khoo KH, Besra GS et al. The pimB gene of Mycobacterium tuberculosis encodes a mannosyltransferase involved in lipoarabinomannan biosynthesis. J Biol Chem, 1999; 274: 31625-31631.

58 Geremia RA, Petroni EA, Ielpi L et al. Towards a classification of glycosyltransferases based on amino acid sequence similarities: prokaryotic $\alpha$-mannosyltransferases. Biochem J, 1996; 318: 133-138.

59 Fernandes ND, Kolattukudy PE. Cloning, sequencing and characterization of a fatty acid synthase-encoding gene from Mycobacterium tuberculosis var. bovis BCG. Gene, 1996; 170: 95-102.

60 Choi KH, Kremer L, Besra GS, Rock CO. Identification and substrate specificity of beta -ketoacyl (acyl carrier protein) synthase III (mtFabH) from Mycobacterium tuberculosis. J Biol Chem, 2000; 275: 28201-28207.

61 Mdluli K, Slayden RA, Zhu Y et al. Inhibition of a Mycobacterium tuberculosis beta-ketoacyl ACP synthase by isoniazid. Science, 1998; 280: 1607-1610.

62 Quemard A, Sacchettini JC, Dessen A et al. Enzymatic characterization of the target for isoniazid in Mycobacterium tuberculosis. Biochemistry, 1995; 34: 8235-8241.

63 Yuan Y, MeadD, Schroeder B Getal. The biosynthesis of mycolic acids in Mycobacterium tuberculosis. Enzymatic methyl(ene)transfer to acyl carrier protein bound meromycolic acid in vitro. J Biol Chem, 1998; 273: 21282-21290.

64 Belisle JT, Vissa VD, Sievert T et al. Role of the major antigen of Mycobacterium tuberculosis in cell wall biogenesis. Science, 1997; 276: 1420-1422.

65 Puech V, Bayan N, Salim K et al. Characterization of the in vivo acceptors of the mycoloyl residues transferred by the corynebacterial PS1 and the related mycobacterial antigens 85. Mol Microbiol, 2000; 35: 1026-1041.

66 Azad AK, Sirakova TD, Fernandes ND, Kolattukudy PE. Gene knockout reveals a novel gene cluster for the synthesis of a class of cell wall lipids unique to pathogenic mycobacteria. J Biol Chem, 1997; 272: 16741-16745.

67 Mathur M, Kolattukudy PE. Molecular cloning and sequencing of the gene for mycocerosic acid synthase, a novel fatty acid elongating multifunctional enzyme, from Mycobacterium tuberculosis var. bovis Bacillus ClametteGuerin. J Biol Chem, 1992; 267: 19388-19395.

68 Camacho LR, Constant P, Raynaud C et al. Analysis of the phthiocerol dimycocerosate locus of Mycobacterium tuberculosis: evidence that this lipid is involved in the cell wall permeability barrier. J Biol Chem, 2001; 276: 19845-19854.

69 Eckstein TM, Silbaq FS, Chatterjee D et al. Identification and recombinant expression of a Mycobacterium avium rhamnosyltransferase gene ( $r t f A)$ involved in glycopeptidolipid biosynthesis. J Bacteriol, 1998; 180: 5567-5573.

70 Wilson VT, Cundliffe E. Characterization and targeted disruption of a glycosyltransferase gene in the tylosin producer, Streptomyces fradiae. Gene, 1998; 214: 95-100.

71 Truman RW, JL Krahenbuhl. Viable M.leprae as a research reagent. Int J Lepr, 2001; 69: 1-12. 